\title{
TRANSPLANTE DE RIM E PÂNCREAS - ANÁLISE DE 55 CASOS DE AGOSTO DE 1987 A JANEIRO DE 2005
}

\author{
Pancreas-kidney transplantation: analysis of 55 cases from 1987 to 2005 \\ Santo Pascual Vitola1, Enilde Eloena Guerra1, Eduardo Chaise Didoné1, Fabian Silva Pires², \\ Jair Garcia da Silva', Valter Duro Garcia ${ }^{3}$, André Ricardo D'Ávila'
}

\section{RESUMO}

Os transplantes de pâncreas e de ilhotas pancreáticas são os únicos métodos terapêuticos capazes de restaurar o estado euglicêmico em pacientes com diabete tipo 1. O transplante pancreático apresentou avanços importantes nos últimos anos; continua, contudo, sendo procedimento cirúrgico complexo, associado a morbimortalidade não desprezível. Objetivo: Relatar a experiência pioneira de nosso serviço com o transplante simultâneo de rim e pâncreas, enfocando os resultados dos últimos 18 anos. Métodos: De agosto de 1987 a janeiro de 2005 foram realizados 53 transplantes simultâneos de pâncreas e rim e 2 de pâncreas após rim. Na primeira fase (1987 a 1998) foram realizados 9 transplantes simultâneos de rim e pâncreas e 1 transplante após rim; na segunda fase (2000-2005) foram realizados 44 transplantes simultâneos de pâncreas e rim e 1 transplante após rim. Resultados: A idade média dos pacientes foi 31,8 anos; 70,9\% estavam em hemodiálise e $20 \%$ em diálise peritonial. A sobrevida atuarial em 2 anos de pacientes e enxertos, renal e pancreático, na segunda fase foram, respectivamente, $81 \%, 77 \%$ e $71 \%$. A análise estatística comparativa mostrou uma melhora na sobrevida do enxerto renal na segunda fase (log-rank=0,03). Conclusão: As mais acuradas avaliações e seleções pré-operatórias dos pacientes, associadas às melhorias na abordagem cirúrgica e aos protocolos de imunossupressão permitiram o alcance de melhores resultados em termos de qualidade de vida e sobrevida para os pacientes diabéticos com insuficiência renal em fase terminal.

Descritores: Transplante de pâncreas, Transplante de órgãos, Diabete mellitus.

\footnotetext{
Trabalho realizado no Serviço de Transplante Renal e Pancreático - Hospital Dom Vicent Scherer - Centro de Transplantes - Complexo Hospitalar Santa Casa de Porto Alegre. Avenida Independência, 155 - Bairro Auxiliadora - CEP 90020-090 - Porto Alegre, Rio Grande do Sul, Brasil.

${ }^{1}$ Cirurgiões da equipe de Transplante de Pâncreas e Rim do Hospital Dom Vicente Scherer - Complexo Hospitalar da Santa Casa de Porto Alegre;

${ }^{2}$ Urologista da equipe de Transplante de Pâncreas e Rim do Hospital Dom Vicente Scherer - Complexo Hospitalar da Santa Casa de Porto Alegre;

${ }^{3}$ Nefrologista. Coordenador da equipe de Transplante de Pâncreas e Rim do Hospital Dom Vicente Scherer - Complexo Hospitalar da Santa Casa de Porto Alegre.

Endereço para correspondência: Dr. André Ricardo D'Avila.Rua Mariante, 288 cj. 706 - Moinhos de Vento. CEP 90430-181 Porto Alegre - Rio Grande do Sul - Brasil E-mail: rdavila@terra.com.br

Recebido em: 30/06/2003 Aceito: 25/11/2004
}

\section{INTRODUÇÃO}

O transplante de pâncreas, assim como o dos demais órgãos sólidos, experimentou uma trajetória crescente de progressos nas áreas de técnica cirúrgica, imunossupressão e cuidados intensivos que permitiram a sua utilização atual em larga escala para a retomada do estado euglicêmico em pacientes diabéticos. Conseqüentemente, o transplante simultâneo de rim e pâncreas (TSRP) livrou-se do estigma de procedimento experimental e tornou-se uma modalidade terapêutica efetiva para o tratamento de pacientes com nefropatia diabética em fase terminal. ${ }^{1,2}$

A melhora obtida nos resultados, associada a um número crescente de indivíduos diabéticos com complicações secundárias limitantes, reflete-se num interesse aumentado por parte de médicos e pacientes em relação a esta modalidade terapêutica. A capacidade de controlar a glicemia, bloquear o avanço das complicações do diabete e a melhora na qualidade de vida proporcionadas faz com que, atualmente, mais de 1200 transplantes de pâncreas sejam feitos a cada ano nos EUA e 240 no Brasil, com números crescentes. ${ }^{3}$ 
O presente estudo revela a experiência pioneira do Complexo Hospitalar Santa Casa (CHSC-RS) nesta modalidade de transplante, com ênfase nos resultados obtidos nos últimos 18 anos. A casuística é apresentada e avaliada de maneira dicotomizada, devido à diferença na morbimortalidade, em função de mudança de técnica e imunossupressão, entre a primeira etapa do programa (1987 a 1998 - 10 primeiros pacientes) e a segunda etapa (de maio de 2000 até janeiro de 2005 - 45 pacientes).

\section{Pacientes e métodos}

O programa de transplante de rim e pâncreas do CHSC-RS iniciouse em agosto de 1987, com o primeiro transplante simultâneo destes órgãos na América do Sul. Desde então até janeiro de 2005, cinqüenta e três TSRP e dois transplantes de pâncreas após rim (TPAR) foram realizados em pacientes portadores de diabete mellitus tipo 1 (insulino-dependente) e em insuficiência renal em fase terminal.

Foram operados 33 pacientes do sexo masculino e 22 do sexo feminino. A idade variou de 17 a 49 anos. Quatro pacientes foram transplantados em período pré-diálise. Dentre os demais, a terapia de reposição renal utilizada em 39 pacientes $(70,9 \%)$ foi a hemodiálise, enquanto a diálise peritonial foi utilizada em apenas 11 pacientes $(20 \%)$.

Durante o período estudado, três diferentes soluções de preservação de órgãos foram empregadas. A solução de Aquatella foi empregada no primeiro caso, em 1987. Nos casos realizados de 1989 a 1993 foram utilizadas as soluções de EuroCollins (5 casos) e solução da Universidade de Wisconsin (UW - um caso). A partir de dezembro de 1993, todos os órgãos foram preservados com a solução UW.

Nos dez primeiros casos, ambos os enxertos foram colocados nas fossas ilíacas por incisões separadas e abordagem extraperitonial. Na fase seguinte, a abordagem foi modificada e os enxertos foram posicionados na cavidade peritonial através de laparotomia mediana.

Foram utilizados enxertos de pâncreas total em todos os pacientes. O primeiro doente foi transplantado apenas com um "botão" de duodeno (justa papilar) que foi anastomosado à bexiga. Nos demais pacientes o enxerto pancreático foi preparado com segmento de duodeno justa papilar, que foi suturado à bexiga ( 9 casos) ou ao intestino delgado (45 casos).

Nos pacientes da primeira fase, a perfusão arterial do enxerto foi feita através de patch de aorta contendo as artérias esplênica e mesentérica superior do doador. Na segunda fase, estes vasos foram seccionados durante a cirurgia de retirada, sendo a reconstrução arterial realizada utilizando-se enxerto em "Y" de artéria ilíaca do doador, transformando assim em único o duplo pedículo arterial. Tal técnica facilita a captação simultânea do fígado e a anastomose arterial no receptor.

Em todos os casos, a reconstrução venosa deu-se com anastomose do remanescente da veia porta na veia ilíaca externa do receptor, sem utilização de enxerto para prolongamento.
Todos os pacientes da primeira fase foram submetidos à drenagem vesical da secreção exócrina através de sutura do duodeno ou de um "botão" de mucosa duodenal à bexiga. Na segunda fase vem sendo utilizada a drenagem entérica, através de anastomose látero-lateral do duodeno com o intestino delgado do receptor.

A profilaxia antimicrobiana foi feita com ampicilina, oxacilina e amicacina em dose única pré-operatória nos primeiros 15 pacientes. Nos demais utilizamos ampicilina-sulbactam e fluconazol por 7 dias.

Para prevenção da trombose vascular, sub-heparinização (5000 UI via subcutânea de $12 / 12 \mathrm{~h}$ por 15 dias) seguida por AAS (200 mg por dia) e dipiridamol (75 mg 3 vezes ao dia) foi o esquema empregado nos casos de 1 a 7. A partir do caso 8, não mais se utilizou dipiridamol e o AAS foi administrado desde o pós-operatório imediato.

Nos pacientes da primeira fase, a imunossupressão utilizada foi o esquema tríplice com ciclosporina, azatioprina e prednisona, com ou sem indução com OKT3. Na segunda fase, os pacientes receberam terapia de indução com OKT3 (um paciente) ou anticorpo anti-receptor IL-2 (basiliximab ou daclizumab). Na terapia de manutenção foram empregados tacrolimus, prednisona e micofenolato ou rapamicina.

Para as análises de sobrevida utilizou-se o cálculo de Kaplan-Meier e o teste de log-rank.

\section{RESULTADOS}

A média de idade foi de 31,8 anos (17 a 49 anos). O tempo médio de diálise foi de 20,2 meses ( 0 a 84 meses) e o tempo médio de diagnóstico do diabete foi de 18,07 anos (8 a 30 anos).

No primeiro caso, em que foi utilizada solução de Acquatella, o pâncreas teve um tempo de isquemia fria de 4 horas e o rim de 8 horas. Nos demais, o tempo de isquemia fria do pâncreas variou de 3,17 a 6 horas (média de 4,16 horas) com a solução de EuroCollins e de 4,72 a 16,33 horas (média de 11,98 horas) com a solução UW. Por outro lado, o tempo de isquemia fria do rim variou de 5,33 a 8,17 horas (com a solução de EuroCollins) e de 7,75 a 19,5 horas (com a solução UW). Para estes últimos intervalos, os tempos médios de isquemia foram, respectivamente, 7,13 e 14,47 horas. Todos os pacientes apresentaram função imediata do enxerto pancreático, ao passo que diurese imediata foi observada em 24 pacientes.

Na primeira fase, a sobrevida dos pacientes em 1 e 2 anos foi de $80 \%$ e $60 \%$. Na segunda fase, a sobrevida cumulativa ao final do $1^{\circ}$ e do $2 \underline{0}$ anos foi de $81 \%$. Em relação à sobrevida do enxerto renal, os resultados na primeira etapa foram de $60 \%$ no primeiro ano e $40 \%$ no segundo. Na etapa seguinte, a sobrevida cumulativa do rim foi de $81 \%$ em 1 ano e $77 \%$ em 2 anos. Quanto ao enxerto pancreático, a sobrevida em 1 ano foi de $60 \%$ e em 2 anos $40 \%$, para os pacientes da primeira fase. Na segunda fase, a sobrevida cumulativa foi de $71 \%$ ao final do $1^{\circ}$ e $2^{\circ}$ anos. A análise comparativa entre as sobrevidas da primeira e segunda fase demonstrou diferença estatisticamente significativa na sobrevida do enxerto renal $(\log$-rank $=0,03)$. 
Na primeira fase, a incidência de fístula pancreática foi de $40 \%$ (4 pacientes), sendo que 3 pacientes evoluíram para óbito por complicações relacionadas às fístulas. Em dois destes pacientes a fístula foi de ocorrência tardia, no $15^{\circ} \mathrm{e} 20^{\circ}$ mês de pós-operatório. Duas pacientes apresentaram trombose do enxerto pancreático. Uma delas, receptora do primeiro transplante de pâncreas após rim, apresentou trombose ileofemoral ipsilateral associada, necessitando enxertectomia e amputação de membro inferior. Após alguns meses de internação evoluiu para óbito por endocardite. A outra paciente está viva com o enxerto renal funcionante. Um paciente necessitou da retirada de ambos os enxertos nos primeiros dias de pósoperatório devido a abscessos peri-pancreáticos e necrose cortical renal. Dois pacientes foram a óbito tardio por intercorrências clínicas (infarto agudo do miocárdio e encefalopatia hepática).

Na fase 2, a mortalidade até o momento é de 17,7\% (8 de 45 pacientes). O seguimento varia de um a 46 meses. Uma paciente evoluiu para morte cerebral de etiologia indeterminada no pósoperatório imediato com enxertos funcionantes. Três pacientes foram a óbito por eventos sépticos: um associado a focos de trombose esplênica no $1^{\circ}$ mês pós-operatório, outro à hemorragia digestiva alta no $3^{\circ}$ mês. $\mathrm{O}$ terceiro paciente evoluiu para sepse e subseqüente óbito, sem fístula demonstrável, no $56^{\circ}$ PO. Fístula duodenal ocorreu em outros três casos, resultando em peritonite difusa e múltiplas reintervenções abdominais. Dois destes pacientes evoluíram para óbito, um associado a coagulopatia de etiologia imunológica e difícil manejo e outro decorrente de sepse. A outra paciente com fístula duodenal foi submetida à enxertectomia e se recuperou com função renal normal. Tromboembolismo pulmonar maciço no $2^{-} \mathrm{PO}$ e hemoperitônio no oitavo PO conduziram ao óbito dois pacientes. Dois pacientes apresentaram coleções intra-abdominais sem maiores repercussões sistêmicas e que foram drenadas satisfatoriamente por punção percutânea. Episódios de suboclusão intestinal, tratados exclusivamente com medidas conservadoras (sondagem gástrica e hidratação IV), foram diagnosticados em outros dois pacientes. Destes, um apresentou hemorragia intestinal baixa no 5 - PO, presumivelmente de origem na anastomose duodenojejunal. Não se evidenciou sangramento ativo na reintervenção. Três pacientes apresentaram trombose do pâncreas, a saber no $2^{\circ}, 19^{\circ}$ e $31^{\circ} \mathrm{PO}$, necessitando de retirada do enxerto, porém com preservação da função renal.

\section{DISCUSSÃO}

Desde o primeiro transplante de pâncreas, realizado por Lillehei em Minneapolis, em 1966, muitas modificações ocorreram em termos de técnica, imunossupressão e cuidados gerais de pós-operatório. A experiência histórica, já demonstrada em literatura, evidencia que os resultados são melhores avaliados quando agrupados em períodos com protocolos semelhantes, pois o impacto resultante da mudança de técnica e/ou manejo clínico é mais evidente. ${ }^{2}$
Na experiência do nosso grupo, pode-se observar a existência de duas fases distintas em termos de resultados, embora sem diferença estatisticamente significativa. Na primeira fase, a elevada taxa de complicações e perda de enxertos ao final do primeiro ano reflete uma associação entre curva de aprendizado e o período de consolidação da técnica e da imunossupressão relacionadas ao procedimento. Os melhores resultados na segunda fase, como o evidenciado na sobrevida do enxerto renal, certamente devem-se aos avanços no manejo dos pacientes no trans e no pós-operatório. A não observância de diferença estatisticamente significativa nas curvas de sobrevida de pacientes e enxerto pancreático entre as duas fases pode se dever ao pouco tempo de seguimento dos últimos pacientes.

A sobrevida similar ao final do $1^{\circ}$ e $2^{\circ} \underline{a}$ anos, na segunda fase, indica a tendência de complicações potencialmente graves surgirem nas primeiras semanas de pós-operatório. De fato, nesta fase virtualmente todas as perdas de pacientes ou enxertos ocorreram até o $3^{\circ}$ mês de pós-operatório. A exceção deve-se à perda de enxerto renal em uma paciente com 1 ano e 4 meses pós transplante, por poliomavírus.

Os tempos médios de isquemia do pâncreas e do rim nas duas fases foram compatíveis com o tipo de solução de preservação utilizada e com a técnica empregada. A presença de função imediata do enxerto pancreático em todos os pacientes reflete ausência de lesão de preservação significativa.

As mudanças na técnica cirúrgica alteraram o padrão das complicações. ${ }^{4,5}$ A incidência de fístula pancreática e suas complicações foram mais graves na primeira fase pelo implante extra-peritonial. Com implante intra-peritonial ocorre absorção das secreções pancreáticas, diminuindo a gravidade das complicações. 6 No entanto, as fístulas oriundas do coto duodenal estão associadas à maior morbidade pela peritonite decorrente. A baixa incidência de trombose do enxerto pancreático na segunda fase ( 3 casos entre 45 ) está relacionada ao aprimoramento da técnica cirúrgica (liberação da veia ilíaca interna) e imunossupressão. Apesar da ocorrência de três casos de fístula duodenal na fase 2 , o emprego de anastomose láterolateral entre o jejuno e o duodeno não parece estar relacionado a uma maior incidência desta complicação. ${ }^{6}$

\section{CONCLUSÃO}

O transplante de pâncreas total e, mais recentemente, o transplante de ilhotas, são as únicas modalidades terapêuticas atualmente disponíveis capazes de restabelecer o controle da glicemia a níveis fisiológicos nos pacientes insulino-dependentes. ${ }^{7}$ Apesar dos avanços obtidos nos últimos anos, o TSRP ainda se trata de procedimento cirúrgico de grande porte, associado a uma significativa morbimortalidade. A avaliação pré-operatória criteriosa dos candidatos, a otimização do seu manejo cirúrgico e o emprego da imunossupressão adequada vem oferecendo a pacientes com diabetes mellitus tipo 1 e nefropatia associada, melhores perspectivas de tempo e qualidade de vida. 


\section{ABSTRACT}

The whole pancreas graft and islet cell transplantation are the only therapeutic options capable to restore euglicemic status in Type 1 diabetic patients. Pancreas transplantation presented a great improvement in the last years, although it continues to be a complex procedure, associated to significant morbidity and mortality. Objective: The authors present their pionnering experience with pancreas transplantation for the last 18 years, emphasizing the results. Method: From 7/1987 to 1/2005 53 simultaneous pancreas/kidney (SPK) and two pancreas after kidney (PAK) transplants have been performed. In the first phase (1987 to 1998) 9 SPK and 1 PAK were performed; in second phase (2000 to 2005), 44 SPK and 1 PAK. Results: Ages ranged from 17 to 49 (mean 31,8). Hemodialysis was the renal replacement therapy in 70,9\% and peritonial dialysis, in $20 \%$. Two-year actuarial survival in second phase was $81 \%, 77 \%$ and $71 \%$ for patients, kidney and pancreas allografts. A statistically significant difference was demonstrated in kidney graft survival between first and second phase patients $(\log$ rank $=0,03)$. Conclusion: The strict preoperative selection of patients, associated to better surgical approach and better immunosuppressive drugs improved either survival as the quality of life of those patients.

Key words: Pancreas transplantation, Organ transplantation, Diabetes mellitus.

\section{REFERÊNCIAS}

Knoll GA, Nichol G. Dialysis, kidney transplantation or pancreas transplantation for patients with diabetes mellitus and renal failure: a decision analysis of treatment options. J Am Soc Nephrol 14: 500-515, 2003.

2. Sutherland DER, Gruessner RWG, Dunn DL et al. Lessons Learned From More Than 1,000 Pancreas Transplants at a Single Institution. Ann Surg 233(4), 2001

3. International Pancreas Transplant Registry 14(1), 2002.
Schulak JA, Henry ML, Munda R et al. Pancreas transplantation in Ohio: a 15-yer outcomes analysis. Surgery 130: 546-53, 2001.

5. Steurer W, Margreiter R, Königsrainer A. Surgical techniques for pancreas transplantation. Acta Chir Austriaca 33(1): 2001.

6. Odorico JS, Sollinger HW. Technical and Immunosuppressive Advances in Transplantation for Insulin-dependent Diabetes Mellitus. World J Surg 26(2), 2002.

. Burridge PW, AMJ Shapiro, Ryan EA et al. Future trends in clinical islet transplantation. Transplantation Proceedings 34: 3347-3348, 2002. 